\title{
Decreased Spontaneous Brain Activity and Functional Connectivity in Type 1 Diabetic Patients Without Microvascular Complications
}

\author{
Wenqing Xia ${ }^{\mathrm{a}} \quad$ Yu-Chen Chen ${ }^{\mathrm{b}} \quad$ Yong Luo $^{\mathrm{a}} \quad$ Dan-Feng Zhang ${ }^{\mathrm{a}}$ Huiyou Chen ${ }^{\mathrm{b}}$ \\ Jianhua Ma $\mathrm{Mindao} \mathrm{Yin}^{\mathrm{b}}$ \\ aDepartment of Endocrinology, Nanjing First Hospital, Nanjing Medical University, Nanjing, \\ bDepartment of Radiology, Nanjing First Hospital, Nanjing Medical University, Nanjing, China
}

\section{Key Words}

Type 1 diabetes mellitus $\cdot$ ALFF $・$ ReHo $\cdot$ Functional connectivity $・$ Resting-state fMRI

\begin{abstract}
Background/Aims: Type 1 diabetes mellitus (T1DM) has been proven to be associated with an increased risk of cognitive dysfunction. In this study, we aimed to investigate whether disrupted spontaneous activity and functional connectivity (FC) exist in T1DM patients using restingstate functional magnetic resonance imaging ( $\mathrm{rs}-\mathrm{fMRI}$ ) and to detect the relationships of these parameters with cognitive impairment. Methods: T1DM patients $(n=35)$ were compared with age-, sex-, and education level-matched healthy controls $(n=50)$ through rs-fMRI. Using rsfMRI professional software, we calculated the amplitude of low-frequency fluctuation (ALFF), regional homogeneity (ReHo), and seed-based FC in the posterior cingulate cortex (PCC) to measure the spontaneous neural activity in the groups. The relationship between rs-fMRI data and cognitive performance was further investigated. Results: Compared with the healthy controls, T1DM patients showed significantly decreased ALFF values in the PCC and right inferior frontal gyrus (IFG), decreased ReHo values in the right middle frontal gyrus (MFG) and reduced FC between the PCC and the right MFG. Furthermore, a positive correlation was found between decreased ALFF values in the PCC and Rey-Osterrieth Complex Figure Test (CFT)delay scores in T1DM patients $(r=0.394, p=0.026)$. Moreover, the Trail Making Test-B (TMT-B) scores showed negative correlations with decreased ReHo values in the right MFG $(r=-0.468$, $p=0.007)$ and reduced FC between the PCC and right MFG $(r=-0.425, p=0.015)$. Conclusion: Our combined analyses revealed decreased spontaneous activity and FC mainly within the default mode network, which was correlated with specific impaired cognitive functioning in T1DM. This study thus elucidates the neurophysiological mechanisms underlying T1DMrelated cognitive impairment and may serve as a reference for future clinical diagnosis.

W. Xia and Y.-C. Chen have contributed equally to this work.




\section{Introduction}

Type 1 diabetes mellitus (T1DM) is a chronic metabolic disease with typical onset in early life and is characterized by absolute insulin deficiency, necessitating exogenous insulin therapy to control blood glucose levels. T1DM patients are exposed to high (hyperglycemia) and low (hypoglycemia) blood glucose levels, and cumulative hyperglycemic exposure can lead to microvascular end-organ damage, such as retinopathy and nephropathy [1]. In addition to microvascular complications, T1DM has been linked with an increased risk of cognitive impairment, which primarily presents as a decrease in information processing speed, attention, and executive function [2-4]. This cognitive decline may be present as early as 2 years after diagnosis and persists well into adulthood and late life $[5,6]$. However, the exact neuropathological mechanism of T1DM-induced cognitive impairment is still largely unclear.

Neuroimaging techniques have been applied to investigate the anatomical and functional alterations in the brain of T1DM patients. Gray matter (GM) atrophy and white matter (WM) lesions, which are common structural abnormalities observed in previous studies, are modestly linked with cognitive decline in T1DM patients [7-12]. Reduced GM volumes have been detected in cortical and subcortical regions of T1DM patients, including occipital, inferior frontal, and parahippocampal regions $[7,11]$. Moreover, low fractional anisotropy in the superior parietal lobule and reduced mean diffusivity in the thalamus have been observed in T1DM patients [10]. However, prior studies predominantly focused on aberrant brain structural changes in T1DM, and very few studies have been performed to explore the effects of T1DM on functional activity.

Resting-state functional magnetic resonance imaging (rs-fMRI) is a powerful tool for evaluating spontaneous neural activity using blood oxygen level-dependent (BOLD) contrast in the absence of tasks with high spatial resolution and easy application [13, 14]. Using rsfMRI, several studies on T1DM have demonstrated disrupted functional connectivity (FC) and network changes that may serve as early biomarkers for subsequent cognitive decline [15-18]. In a seed-based analysis, van Duinkerken et al. found abnormal FC in the subgenual cingulate cortex that was related to cognitive dysfunction in T1DM patients [16]. Moreover, they demonstrated that subcortical eigenvector centrality and degree centrality were lower and bilateral occipital eigenvector centrality was higher in T1DM patients without proliferative retinopathy [18]. Furthermore, through independent component analysis or graph theoretical analysis, T1DM was observed to be associated with disrupted FC in multiple networks involving attention, working memory, auditory, language and processing and the default mode network (DMN) $[15,18,19]$. Among these networks, the DMN, consisting of nodes in the posterior cingulate cortex (PCC)/precuneus, anterior cingulate cortex (ACC), medial prefrontal gyrus (mPFC) and inferior parietal lobule (IPL), is the most active during resting and shows reduced activity when a subject enters a task-based state involving attention or goal-directed behavior, and these DMN activities may be used to predict cognitive impairment $[14,20]$. As the pivotal region of DMN, the PCC reveals diverse cognitive functions including visuospatial memory and emotional processing [21, 22]. During cognitive processing, the PCC is functionally linked with several DMN-related regions, such as the ACC and mPFC [23]. Thus, cognitive impairment in T1DM patients may be related to the FC abnormalities of PCC. To date, the direct relationship between alterations in the PCC or DMN and T1DM-related cognitive impairment remains unclear.

As the effects of hyperglycemia on the brain may be global, a whole-brain analysis of neural function would likely identify other central processing deficits linked with T1DM. The amplitude of low-frequency fluctuation (ALFF) and regional homogeneity (ReHo) are two major data-driven algorithms for the local measurement of spontaneous neural activity. The ALFF measures the amplitude of very low-frequency oscillations in the BOLD signal at the single-voxel level [24], while ReHo analyzes the local neural synchronization of a given voxel with its adjacent voxels [25]. ALFF may be more useful than ReHo for investigating global changes in spontaneous neural activity. On the other hand, ReHo may be more sensitive at 
detecting regional brain abnormalities than ALFF. The combination of the ALFF and ReHo may provide a more comprehensive pathophysiological assessment of human brain dysfunctions than either method alone or other combinations [26-28], indicating that the coexisting neural intensity and coherence abnormalities in specific brain regions may represent more severe brain changes than those revealed by a single method. Thus, we utilized these two common methods as well as FC analysis to identify whole-brain spontaneous neural activity and connectivity in T1DM.

Based on prior work and theoretical considerations, we aimed to combine the ALFF, ReHo, and FC to explore resting-state brain activity in T1DM patients and hypothesized that aberrant ALFF, ReHo, and FC values would be identified mainly within the DMN and would be correlated with specific cognitive performance deficits.

\section{Materials and Methods}

\section{Subjects}

Thirty-five T1DM patients from the Department of Endocrinology, Nanjing First Hospital, and 50 healthy controls (aged between 40 and 70 years, all right-handed and with at least 6 years of education) were recruited through online advertisements and matched for age, sex and education level between June 2017 and June 2018. Participants were excluded from the current study if they met the criteria for mild cognitive impairment as described by Petersen [29].

No participants were excluded from the fMRI analysis because of excessive head motion during scanning. Participants were excluded from the study if they acknowledged a history of known stroke, alcoholism, Parkinson's disease, epilepsy, traumatic brain injury, and major medical conditions (e.g., cancer, thyroid dysfunction, severe heart diseases, damaged liver or kidney function). The fasting glucose levels and postprandial glucose levels were measured in healthy controls, and any individual with a fasting glucose level $>6.1 \mathrm{mmol} / \mathrm{l}$ or postprandial glucose level $>7.8 \mathrm{mmol} / \mathrm{l}$ was excluded.

Patients with macrovascular diseases or clinically detectable microvascular complications (e.g., retinopathy, nephropathy, neuropathy) were also excluded. Retinopathy was graded according to the Wisconsin Epidemiologic Study [30]. Diabetic nephropathy was assessed using microalbuminuria, which was defined as an albumin-to-creatinine ratio $>2.5 \mathrm{mg} / \mathrm{mmol}$ for males and $>3.5 \mathrm{mg} / \mathrm{mmol}$ for females. The presence of peripheral neuropathy was based on the results of an annual checkup of the patients.

\section{Clinical data and neuropsychological tests}

The demographic characteristics of each subject were obtained. Blood samples were collected by venipuncture at $8 \mathrm{AM}$ after an overnight fast. The levels of fasting blood glucose, HbA1c, and blood lipids were measured.

Objective cognitive assessment, including global cognitive tests and an extensive neuropsychological test battery, were administered to evaluate the neuropsychological state of each individual. Global cognitive tests contained the Mini Mental State Exam (MMSE) and the Montreal Cognitive Assessment (MoCA). The battery of neuropsychological tests included the Auditory Verbal Learning Test (AVLT), Rey-Osterrieth Complex Figure Test (CFT), Digit Span Test (DST), Trail Making Test-A and B (TMT-A and TMT-B), Clock Drawing Test (CDT), Verbal Fluency Test (VFT), and Digit Symbol Substitution Test (DSST).

\section{MRI data acquisition}

The subjects were scanned using a 3.0 T MRI scanner (Ingenia, Philips Medical Systems, Netherlands) with an 8-channel receiver array head coil. Subjects lay supine with their head fixed by foam pads and a belt to minimize head motion. And earplugs were used to reduce scanner noise for the subject. The subjects were instructed to lie quietly with their eyes closed but not to fall asleep, not to think of anything in particular, and to avoid head motion during the functional MR imaging. Structural images were acquired using a three-dimensional turbo fast echo (3D-TFE) T1WI sequence at a high resolution using the following parameters: repetition time $(\mathrm{TR}) /$ echo time $(\mathrm{TE})=8.1 / 3.7 \mathrm{~ms}$; slices=170; thickness=1 $\mathrm{mm}$; gap=0 mm; flip angle $(\mathrm{FA})=8^{\circ}$; acquisition matrix $=256 \times 256$; field of view $(\mathrm{FOV})=256 \mathrm{~mm} \times 256 \mathrm{~mm}$. Fluid-attenuated inversion recovery (FLAIR) scans were also acquired with the following parameters: TR/TE=7000/120 


\section{Cellular Physiology Cell Physiol Biochem 2018;51:2694-2703

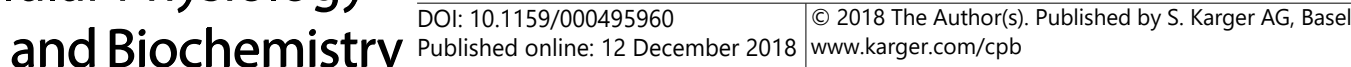

Xia et al.: Decreased Neural Activity in Type 1 Diabetes

$\mathrm{ms}$; slices $=18$; thickness $=6 \mathrm{~mm}$; gap $=1.3 \mathrm{~mm} ; \mathrm{FA}=110^{\circ}$; and voxel size $=0.65 \times 0.95 \times 6 \mathrm{~mm}^{3}$. The functional images were obtained axially using a gradient echo-planar imaging sequence as follows: TR/TE $=2000 / 30$ $\mathrm{ms}$; slices=36; thickness $=4 \mathrm{~mm}$; gap $=0 \mathrm{~mm} ; \mathrm{FOV}=240 \mathrm{~mm} \times 240 \mathrm{~mm}$; acquisition matrix $=64 \times 64 ; \mathrm{FA}=90^{\circ}$; and voxel size $=3.75 \times 3.75 \times 4.0 \mathrm{~mm}^{3}$. The SENSE is used for parallel imaging.

\section{Functional data preprocessing}

Data analyses were conducted using Data Processing \& Analysis for (Resting-State) Brain Imaging (DPABI_V2.3_170105)[31] using the following stages: 240 volumes were scanned, and the first 10 volumes were discarded, followed by a slice-timing adjustment, realignment for head-motion correction, and spatial normalization to the Montreal Neurological Institute (MNI) template (resampling voxel size $=3 \times 3 \times 3$ $\mathrm{mm}^{3}$ ) in addition to smoothing with an isotropic Gaussian kernel (FWHM=6 mm), detrend and filtering $(0.01-0.08 \mathrm{~Hz})$. Participants with a head motion $>2.0 \mathrm{~mm}$ translation or a $2.0^{\circ}$ rotation in any direction were excluded to minimize movement artifacts.

\section{ALFF and ReHo analyses}

ALFF analysis was conducted using the REST software through the procedure described in previous studies [24]. Images were first smoothed with a Gaussian kernel of $6 \mathrm{~mm}$ full-width at half maximum (FWHM). Next, the time series were transformed to the frequency domain using a fast Fourier Transform. The square root of the power spectrum was then computed and averaged across 0.01-0.08 Hz within each voxel. The averaged square root was taken as the ALFF. For standardization purposes, the ALFF of each voxel was divided by the global mean ALFF value.

ReHo was also calculated using the REST software through the procedure described in previous studies [25]. Images were analyzed to calculate Kendall's coefficient of concordance of the time courses of a given voxel with its twenty-six nearest neighboring voxels. The ReHo maps were then spatially smoothed with a Gaussian kernel of $6 \mathrm{~mm}$. The ReHo value of each voxel was standardized by dividing the raw value by the global mean ReHo value that was acquired with a computation method similar to that used to calculate the global mean ALFF value.

\section{FC analyses}

The resting-state FC was calculated using the REST software. The seed regions of interest (ROIs) of the PCC were generated using the WFU_PickAtlas Version 3.0.5 (http://fmri.wfubmc.edu/software/PickAtlas) [32]. The mean time series of the PCC was acquired for use as a reference time course. Then, Pearson's correlation coefficients were computed between the mean signal change in the ROI (PCC) and the time series of each voxel. Finally, correlation coefficients were converted to $\mathrm{Z}$ values using Fisher's z-transformation to standardize the statistical analysis. Six head motion parameters and a mean time series of global, WM and cerebrospinal fluid (CSF) signals were included as confounding factors in the regression analysis to remove their possible effects on the results.

\section{Statistical analyses}

Demographic variables and cognitive performance scores were compared between T1DM patients and healthy controls using SPSS 18.0 (SPSS, Inc., Chicago, IL). An independent two-sample $t$-test was used for continuous variables, and the $\chi 2$ test was used for proportions. Values of $p<0.05$ were considered statistically significant.

For group analyses, the individual ALFF, ReHo, and FC maps were computed in a voxel-wise manner for T1DM patients and healthy controls. Significant thresholds were corrected using false discovery rate (FDR) criterion and set at $\mathrm{p}<0.01$. These maps were entered into a voxel-wise two-sample $t$-test to investigate the differences in ALFF, ReHo, and FC between T1DM patients and healthy controls using a default whole-brain mask. Age, sex, and education level were entered as nuisance covariates. Multiple comparison correction was performed using cluster-level inference at $\mathrm{p}<0.01$ corrected by family-wise error (FWE).

To investigate the relationship between ALFF, ReHo, and FC values of the peak voxels and each clinical characteristic and cognitive performance of T1DM patients, we performed Pearson's correlation analyses using the SPSS software. Partial correlations were analyzed using age, sex, and education level as covariates. A value of $p<0.05$ was considered statistically significant. Bonferroni correction was used for multiple comparisons in the correlation analyses. 


\section{Results}

Clinical and neuropsychological data

The demographic and characteristic data of the participants are shown in Table 1. The two groups did not differ significantly in age, sex, education level, body mass index (BMI), blood pressure, triglycerides, and smoking history. As shown in Table 2, T1DM patients had significantly worse CFT-delay and TMT-B score than healthy controls $(p<0.05)$. However, the other neuropsychological tests showed slight but nonsignificant decrease in cognitive performance in the T1DM patients $(p>0.05)$.

\section{MRI data}

From group analyses, significantly stronger ALFF, ReHo and FC values were found mainly within the DMN regions, including the PCC, precuneus, middle frontal gyrus (MFG), and IPL, in both T1DM patients and healthy controls. Compared with the healthy controls, T1DM patients had significantly decreased ALFF values in the PCC and right inferior frontal gyrus (IFG) and decreased ReHo values in the right MFG (Fig. 1A-B, Table 3). Moreover, relative to the healthy controls, T1DM patients exhibited reduced FC between the PCC and right MFG (Fig. 1C, Table 3). In addition, there were no significant structural changes between T1DM patients and healthy controls.

\section{Correlation analysis}

After correcting for age, sex, and education level, we found a positive correlation between the decreased ALFF values in the PCC and CFT-delay scores in T1DM patients $(r=0.394$, $p=0.026$ ) (Fig. 2A). Moreover, the TMT-B scores showed negative correlations with decreased ReHo values in the right MFG ( $r=-0.468, p=0.007)$ (Fig. 2B) and reduced FC between the PCC and right MFG ( $r=-0.425, p=0.015$ ) (Fig. 2C). However, no significant correlations persisted after Bonferroni correction, probably partly due to the relatively strict calculation. No such associations were detected in the healthy control group. The decreased neural activity was independent of other clinical characteristics and cognitive tests. 
Fig. 1. (A) Compared with the healthy controls, type 1 diabetes mellitus (T1DM) patients showed significantly decreased amplitude of low-frequency fluctuation (ALFF) values in the posterior cingulate cortex (PCC) and right inferior frontal gyrus (IFG) $(\mathrm{p}<0.01$ corrected by AlphaSim); (B) Compared with the healthy controls, T1DM patients showed significantly decreased regional homogeneity (ReHo) values in the right middle frontal gyrus (MFG) $(\mathrm{p}<0.01$ corrected by AlphaSim); (C) Relative to the healthy controls, T1DM patients exhibited reduced FC between the PCC and the right MFG $(\mathrm{p}<0.01$ corrected by AlphaSim). The left side of the image

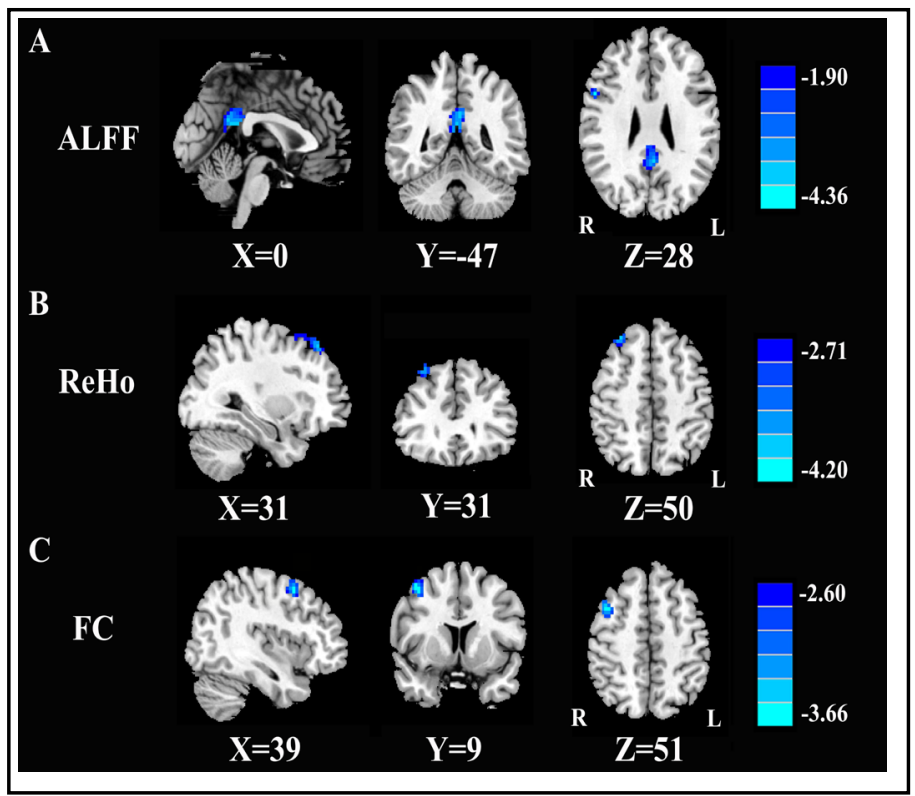
corresponds to the right hemisphere.

Table 3. Decreased spontaneous activity and functional connectivity in T1DM patients compared with healthy controls. A corrected threshold of $\mathrm{p}<0.01$ with AlphaSim correction. Age, gender, and education were used as nuisance covariates. BA, Brodmann's area; MNI, Montreal Neurological Institute; R, right

\begin{tabular}{lcccc}
\hline Brain regions & BA & MNI Coordinates $\mathrm{x}, \mathrm{y}, \mathrm{z}(\mathrm{mm})$ & Peak T value & Voxels \\
\hline ALFF & & & & \\
Posterior cingulate cortex & 31 & $0,-47,28$ & -3.7489 & 135 \\
R inferior frontal gyrus & 9 & $54,9,28$ & -4.2180 & 40 \\
ReHo R middle frontal gyrus & 8 & $31,31,50$ & -3.7630 & 58 \\
FC R middle frontal gyrus & 6 & $39,9,51$ & -3.8883 & 48 \\
\hline
\end{tabular}
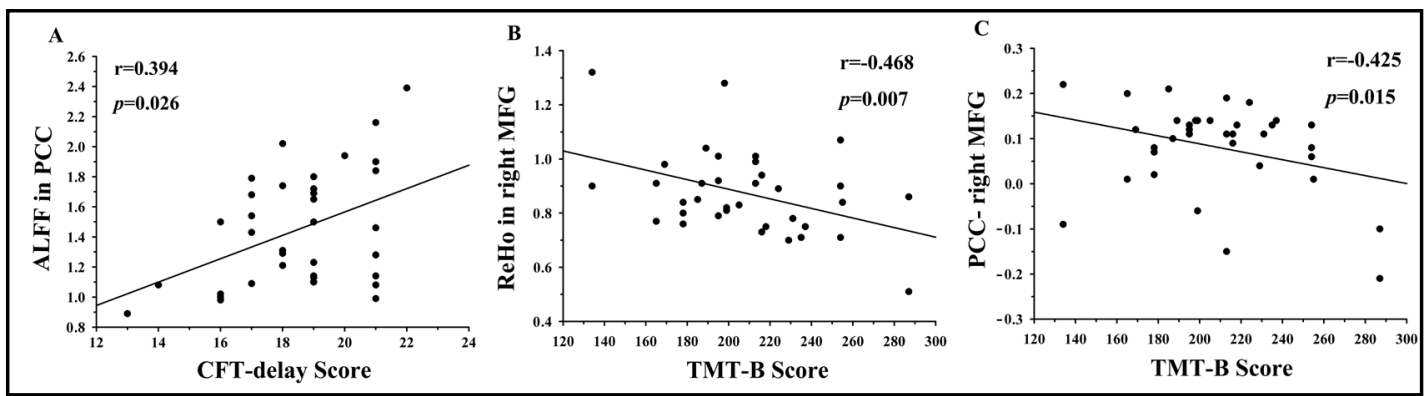

Fig. 2. (A) Positive correlations between decreased ALFF values in the PCC and CFT-delay scores in T1DM patients ( $r=0.394, p=0.026$ ); (B) Negative correlations between decreased ReHo values in the right MFG and TMT-B scores in T1DM patients ( $r=-0.468, p=0.007)$; (C) Negative correlations of reduced FC between the PCC and right MFG with TMT-B scores in T1DM patients ( $r=-0.425, \mathrm{p}=0.015)$.

\section{Discussion}

To the best of our knowledge, the current study is the first to examine abnormal neuronal activity and cognitive impairment in Asian patients with T1DM. Compared with those in healthy controls, we found impaired cognitive function and decreased spontaneous brain activity and FC within the DMN in T1DM patients. Moreover, we focused on the correlation between rs-fMRI data and specific cognitive domains. This study may lead to a better understanding of the neurological pathophysiology in T1DM patients. Our results suggested 
that abnormal alterations in the DMN may play a key role in T1DM-related cognitive dysfunction and serve as a guide for clinical diagnosis. Most importantly, rs-fMRI may be able to track the very early progression of brain functional alterations associated with T1DM, prior to the onset of aberrant cognitive performance. Nevertheless, more comprehensive research should be performed to confirm these implications.

In this study, multidimensional neuropsychological tests were conducted to evaluate the cognitive function of each individual. However, except for the CFT delay and TMT-B, most tests showed nonsignificant differences between T1DM patients and healthy controls. The CFT-delay and TMT-B scores in the T1DM group showed significant decreasing trends compared with the scores in the control group, suggesting that hyperglycemia may lead to overt damage to memory and executive functions in T1DM patients.

The ALFF and ReHo have been widely used to explore the potential pathogenesis of various neuropsychiatric diseases, especially cognition-related diseases [24-28, 33, 34]. These two parameters are based on different neurophysiological mechanisms, with ALFF measuring neural intensity [24] and ReHo representing neural coherence [25]. In the current study, decreased ALFF and ReHo were detected in the PCC and prefrontal cortex. Similarly, reduced ALFF and ReHo have been observed in the same brain regions in patients with type 2 diabetes mellitus (T2DM)[27]. Thus, we infer that T1DM and T2DM patients may share the similar mechanisms of cognitive changes in the brain. Besides, we propose that the coexisting abnormalities in neural intensity and coherence in these specific brain regions may represent more severe brain changes than those revealed by single changes.

Abnormalities in the DMN have been documented in T1DM patients $[16,19]$. Lower FC within the DMN was correlated with poorer cognitive functioning in T1DM patients with microvascular complications [19]. However, our results showed decreased spontaneous activity and FC within DMN regions associated with poor cognitive performance in T1DM patients without microvascular complications. We thus infer that the DMN may be vulnerable to T1DM at the early stage in the absence of microvascular complications. Within the DMN, the PCC functions as the central node and is a highly connected and metabolically active region in the brain [35]. This region is involved in memory, emotion, and intrinsic control networks. Decreased spontaneous neural activity in the PCC may have thus contributed to the poor performance of the patients on CFT. Recently, van Duinkerken et al. observed a decrease in executive functions in T1DM patients, which was accompanied by an accelerated loss of the PCC volume [36]. However, no study has observed decreased functional activity in the PCC of T1DM patients. Interestingly, prior studies have demonstrated reduced resting-state neural activity in the PCC in T2DM patients [37-39]. We speculate that T1DM and T2DM also share neuropathological mechanisms in the PCC region. Nevertheless, this region-specific neural cognition relationship may support our hypothesis that neural activity abnormalities play a pivotal role in T1DM-related cognitive impairment.

The prefrontal cortex is involved in emotional processing and executive functioning [40]. We detected decreased spontaneous neural activity and FC in the prefrontal cortex (especially the MFG), which was correlated with poor TMT-B performance. The TMT-B score reflects the function of the prefrontal cortex and is a neurocognitive parameter that is commonly used to define T1DM-related cognitive dysfunction. According to previous structural and functional MRI studies involving T1DM patients, the structure and function of the prefrontal cortex are typically impaired in these populations $[15,16,36,41,42]$, supporting the results of our correlation analysis in which the hypoconnectivity of the MFG in T1DM patients was associated with the TMT-B score. In line with prior findings, our results have implications for understanding the specific role of abnormalities in the MFG of T1DM patients and indicate that the prefrontal cortex may provide valuable insights into the neural mechanisms underlying T1DM.

Nevertheless, several limitations in our study must be acknowledged. First, we admit that it is difficult to make direct causal inferences regarding the relationships between abnormal neural activity and cognitive impairment in T1DM patients considering the cross-sectional nature of our experimental design and limited sample size. Thus, further 
longitudinal studies involving a larger number of subjects are required to confirm the present conclusions. Second, there are currently no diagnostic criteria for T1DM-related cognitive impairment, and this lack of objective and specific neurocognitive assessment limited our interpretation of the results. Third, the subjects in this study did not match on other variations like IQ or psychiatric problems, which could affect the neuropsychological function. These variations need to be considered in our future study. Moreover, we selected the PCC as the only seed region to investigate the intrinsic FC patterns in the DMN of T1DM patients. The current seed-based approach could be extended to other DMN regions, such as the anterior cingulate cortex (ACC), IPL, and precuneus. Additionally, more studies are required to obtain structural evidence, such as data from diffusion tensor imaging (DTI), to demonstrate the basis of functional modulation within the DMN. Finally, several potential confounding factors, such as blood glucose variations during MR scan, aortic macrovascular disease, ApoEs4 and other T1DM-related genotypes, could not be completely eliminated in the current study. Thus, more studies are required in this field to derive more compelling conclusions.

\section{Conclusion}

Our combined ALFF, ReHo, and FC analyses revealed decreased spontaneous brain activity and connectivity mainly in the PCC and prefrontal cortex, and this result was correlated with specific impaired cognitive functioning in T1DM patients without microvascular complications. This study provides new insights and enhances our understanding of brain abnormalities and their relationships with T1DM-related cognitive impairment. Most importantly, the results suggest that rs-fMRI may be used to track the very early progression of brain functional alterations associated with T1DM.

\section{Acknowledgements}

We would like to express our heartfelt gratitude to all the staff of the Department of Endocrinology and the Department of Radiology, Nanjing First Hospital, Nanjing Medical University, for their selfless help and valuable assistance. This work was supported by the Natural Science Foundation of Jiangsu Higher Education Institutions (No. 18KJB320007), Medical Science and Technology Development Foundation of Nanjing Department of Health (No. YKK16140), Jiangsu Provincial Special Program of Medical Science (BE2017614), Youth Medical Talents of Jiangsu Province (No. QNRC2016062), and 14th "Six Talent Peaks" Project of Jiangsu Province (No. YY-079). WX and Y-C C designed the experiment, collected the data, performed the analysis and wrote the manuscript. YL, D-F Z, and HC collected the data. XY and $\mathrm{J}-\mathrm{H} \mathrm{M}$ contributed to the discussion and manuscript revision. All subjects provided written informed consent before their participation in the study protocol, which was approved by the Research Ethics Committee of Nanjing Medical University.

\section{Disclosure Statement}

The authors declare that there is no potential conflict regarding the publication of this paper. 


\section{Cellular Physiology Cell Physiol Biochem 2018;51:2694-2703 and Biochemistry \begin{tabular}{l|l} 
DOI: 10.1159/000495960 & $\begin{array}{l}\text { (c) } 2018 \text { The Author(s). Published by S. Karger AG, Basel } \\
\text { www.karger.com/cpb }\end{array}$
\end{tabular}}

Xia et al.: Decreased Neural Activity in Type 1 Diabetes

\section{References}

1 Brownlee M: The pathobiology of diabetic complications: a unifying mechanism. Diabetes 2005;54:16151625.

2 Brands AM, Biessels GJ, De Haan EH, Kappelle LJ, Kessels RP: The effects of type 1 diabetes on cognitive performance: a meta-analysis. Diabetes care 2005;28:726-735.

3 Broadley MM, White MJ, Andrew B: A Systematic Review and Meta-analysis of Executive Function Performance in Type 1 Diabetes Mellitus. Psychosom Med 2017;79:684-696.

-4 Gaudieri PA, Chen R, Greer TF, Holmes CS: Cognitive function in children with type 1 diabetes: a metaanalysis. Diabetes care 2008;31:1892-1897.

-5 Brands AM, Kessels RP, Hoogma RP, Henselmans JM, van der Beek Boter JW, Kappelle LJ, de Haan EH, Biessels GJ: Cognitive performance, psychological well-being, and brain magnetic resonance imaging in older patients with type 1 diabetes. Diabetes 2006;55:1800-1806.

6 Northam EA, Anderson PJ, Werther GA, Warne GL, Adler RG, Andrewes D: Neuropsychological complications of IDDM in children 2 years after disease onset. Diabetes care 1998;21:379-384.

7 Musen G, Lyoo IK, Sparks CR, Weinger K, Hwang J, Ryan CM, Jimerson DC, Hennen J, Renshaw $\mathrm{PF}$, Jacobson AM: Effects of type 1 diabetes on gray matter density as measured by voxel-based morphometry. Diabetes 2006;55:326-333.

8 Lyoo IK, Yoon S, Renshaw PF, Hwang J, Bae S, Musen G, Kim JE, Bolo N, Jeong HS, Simonson DC: Networklevel structural abnormalities of cerebral cortex in type 1 diabetes mellitus. PLoS One 2013;8:e71304.

-9 van Duinkerken E, Ijzerman RG, Klein M, Moll AC, Snoek FJ, Scheltens P, Pouwels PJ, Barkhof F, Diamant M, Tijms BM: Disrupted subject-specific gray matter network properties and cognitive dysfunction in type 1 diabetes patients with and without proliferative retinopathy. Hum Brain Mapp 2016;37:11941208.

10 Antenor-Dorsey JAV, Meyer E, Rutlin J, Perantie DC, White NH, Arbelaez AM, Shimony JS, Hershey T: White matter microstructural integrity in youth with type 1 diabetes. Diabetes 2013;62:581-589.

-11 Northam EA, Rankins D, Lin A, Wellard RM, Pell GS, Finch SJ, Werther GA, Cameron FJ: Central nervous system function in youth with type 1 diabetes 12 years after disease onset. Diabetes care 2009;32:445450 .

12 Nunley KA, Ryan CM, Orchard TJ, Aizenstein HJ, Jennings JR, Ryan J, Zgibor JC, Boudreau RM, Costacou T, Maynard JD: White matter hyperintensities in middle-aged adults with childhood-onset type 1 diabetes. Neurology 2015;84:2062-2069.

13 Biswal B, Zerrin Yetkin F, Haughton VM, Hyde JS: Functional connectivity in the motor cortex of resting human brain using echo-planar mri. Magn Reson Med 1995;34:537-541.

14 Raichle ME, MacLeod AM, Snyder AZ, Powers WJ, Gusnard DA, Shulman GL: A default mode of brain function. Proc. Natl. Acad. Sci. U. S. A. 2001;98:676-682.

-15 Van Duinkerken E, Schoonheim MM, Sanz-Arigita EJ, IJzerman RG, Moll AC, Snoek FJ, Ryan CM, Klein M, Diamant M, Barkhof F: Resting-state brain networks in type 1 diabetic patients with and without microangiopathy and their relation to cognitive functions and disease variables. Diabetes 2012;61:18141821.

-16 van Duinkerken E, Ryan CM, Schoonheim MM, Barkhof F, Klein M, Moll AC, Diamant M, IJzerman RG, Snoek FJ: Subgenual cingulate cortex functional connectivity in relation to depressive symptoms and cognitive functioning in type 1 diabetes mellitus patients. Psychosom Med 2016;78:740-749.

17 Ryan JP, Aizenstein HJ, Orchard TJ, Ryan CM, Saxton JA, Fine DF, Nunley KA, Rosano C: Age of childhood onset in type 1 diabetes and functional brain connectivity in midlife. Psychosom Med 2015;77:622.

18 van Duinkerken E, Schoonheim MM, IJzerman RG, Moll AC, Landeira-Fernandez J, Klein M, Diamant M, Snoek FJ, Barkhof F, Wink AM: Altered eigenvector centrality is related to local resting-state network functional connectivity in patients with longstanding type 1 diabetes mellitus. Hum Brain Mapp 2017;38:3623-3636.

19 Demuru M, van Duinkerken E, Fraschini M, Marrosu F, Snoek FJ, Barkhof F, Klein M, Diamant M, Hillebrand A: Changes in MEG resting-state networks are related to cognitive decline in type 1 diabetes mellitus patients. Neuroimage Clin 2014;5:69-76.

-20 Buckner RL, Andrews-Hanna JR, Schacter DL: The brain's default network. Ann N Y Acad Sci 2008;1124:1-38. 


\section{Cellular Physiology Cell Physiol Biochem 2018;51:2694-2703 and Biochemistry \begin{tabular}{l|l}
$\overline{D O I}$ Published 10.1159/000495960 & $\begin{array}{l}\text { (c) 2018 The Author(s). Published by S. Karger AG, Basel } \\
\text { www.karger.com/cpb }\end{array}$
\end{tabular}}

Xia et al.: Decreased Neural Activity in Type 1 Diabetes

21 Leech R, Sharp DJ: The role of the posterior cingulate cortex in cognition and disease. Brain 2014;137:1232.

-22 Sestieri C, Shulman GL, Corbetta M: The contribution of the human posterior parietal cortex to episodic memory. Nat Rev Neurosci 2017;18:183-192.

23 Fransson P, Marrelec G: The precuneus/posterior cingulate cortex plays a pivotal role in the default mode network: Evidence from a partial correlation network analysis. NeuroImage 2008;42:1178-1184.

-24 Zang YF, He Y, Zhu CZ, Cao QJ, Sui MQ, Liang M, Tian LX, Jiang TZ, Wang YF: Altered baseline brain activity in children with ADHD revealed by resting-state functional MRI. Brain Dev 2007;29:83-91.

25 Zang Y, Jiang T, Lu Y, He Y, Tian L: Regional homogeneity approach to fMRI data analysis. NeuroImage 2004;22:394-400.

-26 Zheng J-X, Chen Y-C, Chen H, Jiang L, Bo F, Feng Y, Tang W-W, Yin X, Gu J-P: Disrupted spontaneous neural activity related to cognitive impairment in postpartum women. Front Psychol 2018;9:624.

27 Cui Y, Jiao Y, Chen Y-C, Wang K, Gao B, Wen S, Ju S, Teng G-J: Altered spontaneous brain activity in type 2 diabetes: a resting-state functional MRI study. Diabetes 2014;63:749-760.

28 Zhang J, Chen Y-C, Feng X, Yang M, Liu B, Qian C, Wang J, Salvi R, Teng G-J: Impairments of thalamic resting-state functional connectivity in patients with chronic tinnitus. Eur J Radiol 2015;84(7):1277-84.

29 Petersen RC: Mild cognitive impairment as a diagnostic entity. J Intern Med 2004;256:183-194.

-30 Klein R, Klein BE, Magli YL, Brothers RJ, Meuer SM, Moss SE, Davis MD: An alternative method of grading diabetic retinopathy. Ophthalmology 1986;93:1183-1187.

-31 Yan C-G, Wang X-D, Zuo X-N, Zang Y-F: DPABI: data processing \& analysis for (resting-state) brain imaging. Neuroinformatics 2016;14:339-351.

-32 Maldjian JA, Laurienti PJ, Kraft RA, Burdette JH: An automated method for neuroanatomic and cytoarchitectonic atlas-based interrogation of fMRI data sets. NeuroImage 2003;19:1233-1239.

-33 An L, Cao Q-J, Sui M-Q, Sun L, Zou Q-H, Zang Y-F, Wang Y-F: Local synchronization and amplitude of the fluctuation of spontaneous brain activity in attention-deficit/hyperactivity disorder: a resting-state fMRI study. Neurosci Bull 2013;29:603-613.

-34 Wang Z, Yan C, Zhao C, Qi Z, Zhou W, Lu J, He Y, Li K: Spatial patterns of intrinsic brain activity in mild cognitive impairment and alzheimer's disease: A resting-state functional MRI study. Hum Brain Mapp 2011;32:1720-1740.

-35 Fransson P, Marrelec G: The precuneus/posterior cingulate cortex plays a pivotal role in the default mode network: Evidence from a partial correlation network analysis. NeuroImage 2008;42:1178-1184.

-36 van Duinkerken E, Steenwijk MD, Klein M, Barkhof F, Mograbi DC, Diamant M, Snoek FJ, Ijzerman RG: Accelerated executive functions decline and gray matter structural changes in middle-aged type 1 diabetes mellitus patients with proliferative retinopathy. J Diabetes 2018;10(11):835-846.

37 Xia W, Chen Y-C, Ma J: Resting-State Brain Anomalies in Type 2 Diabetes: A Meta-Analysis. Front Aging Neurosci 2017;9:14.

38 Musen G, Jacobson AM, Bolo NR, Simonson DC, Shenton ME, McCartney RL, Flores VL, Hoogenboom WS: Resting-state brain functional connectivity is altered in type 2 diabetes. Diabetes 2012;61:2375-2379.

-39 Chen Y-C, Jiao Y, Cui Y, Shang S-A, Ding J, Feng Y, Song W, Ju S-H, Teng G-J: Aberrant brain functional connectivity related to insulin resistance in type 2 diabetes: a resting-state fMRI study. Diabetes care 2014;37:1689-1696.

40 Alvarez JA, Emory E: Executive function and the frontal lobes: a meta-analytic review. Neuropsychol Rev 2006;16:17-42.

41 Lyoo IK, Yoon S, Jacobson AM, Hwang J, Musen G, Kim JE, Simonson DC, Bae S, Bolo N, Kim DJ: Prefrontal cortical deficits in type 1 diabetes mellitus: brain correlates of comorbid depression. Arch Gen Psychiatry 2012;69:1267-1276.

42 Moulton CD, Costafreda SG, Horton P, Ismail K, Fu CH: Meta-analyses of structural regional cerebral effects in type 1 and type 2 diabetes. Brain Imaging Behav 2015;9:651-662. 